\title{
THE RESPONSE TO A HOT SPOT IN A COMBUSTION PROBLEM
}

\author{
K. K. TAM and M. T. KIANG
}

(Received 6 May 1980)

(Revised 16 October 1980)

\begin{abstract}
A simple model for a problem in combustion theory has multiple steady state solutions when a parameter is in a certain range. This note deals with the initial value problem when the initial temperature takes the form of a hot spot. Estimates on the extent and temperature of the spot for the steady state solution to be super-critical are obtained.
\end{abstract}

\section{Introduction}

A simple model for a problem in combustion theory is (see [3])

$$
\begin{gathered}
\frac{\partial T}{\partial t}=\nabla^{2} T+\delta \exp (\alpha T /(\alpha+T)) \text { in } D \times\{t: t>0\}, \\
T(\mathbf{x}, 0)=h(\mathbf{x}) \quad \text { and } \quad T=0 \text { on } \partial D,
\end{gathered}
$$

where $T, \mathbf{x}$ and $t$ are respectively the dimensionless temperature, spatial and time variables, $\delta$ a parameter and $\alpha$ is a constant with magnitude greater than 4 (see [7]). This problem has been considered by a number of authors, [3], [4] and [5] among others. It is known that when $\delta$ is within a certain range, say $0<\delta_{e}<\delta<\delta_{\text {cr }}$, equation (1) has two stable steady state solutions: a sub-critical solution in which the temperature is of order one, and a super-critical solution in which the temperature is exponentially large. Estimates of $\delta_{e}$ and $\delta_{\mathrm{cr}}$, as well as the influence of the initial data on the attainment of super-critical state were considered in [6], where $T$ was assumed to depend only on the radial distance $r$ and time $t$, when $D$ is a sphere or a cylinder.

OCopyright Australian Mathematical Society 1981 
In this note, we extend the results in [6] for the case where the domain $D$ is a sphere, and investigate the response of equation (1) to a hot spot when $\delta_{e}<\delta<$ $\delta_{\mathrm{cr}}$. In particular, we want to estimate the extent and temperature of the hot spot for equation (1) to reach a super-critical state.

\section{The initial value problem}

Let $(r, \theta, \psi)$ be spherical coordinates, and the domain described by $0<r<1$, $0 \leqslant \theta \leqslant \pi, 0 \leqslant \psi<2 \pi$. At $t=0$, let there be a hot spot with extent described by

$T(\mathbf{x}, 0)=T_{0}(r, \theta)=\left\{\begin{array}{ll}A & \text { for } r_{0}-\beta \varepsilon<r<r_{0}<1,0<\theta<\nu \varepsilon, 0<\psi<2 \pi, \\ 0 & \text { elsewhere }\end{array}\right\}$

where $\beta, \nu$ are constants, and $\varepsilon=\exp (-\alpha)$. Because of the choice of the location of the hot spot, we can assume the temperature $T$ to be independent of the angle $\psi$.

We rewrite equation (1) as an integral equation

$$
\begin{aligned}
T(P, t)= & \int_{D} G(P, Q, T) T_{0}(Q) d V_{Q} \\
& +\delta \int_{0}^{t} \int_{D} G(P, Q, t-\tau) \exp \left(\frac{\alpha T(Q, \tau)}{\alpha+T(Q, \tau)}\right) d V_{Q} d \tau,
\end{aligned}
$$

where $G$ is the Green's function for the operator $\left((\partial / \partial t)-\nabla^{2}\right)$, with homogeneous initial and boundary conditions and $P, Q$ denote the field point and source point with coordinates $(r, \theta, \psi)$ and $\left(r^{\prime}, \theta^{\prime}, \psi^{\prime}\right)$, respectively. We have

$$
\begin{aligned}
G(P, Q, t)= & \frac{1}{2 \pi \sqrt{r r^{\prime}}} \sum_{\substack{n=0, p=1}}^{\infty} \frac{(2 n+1) J_{n+1 / 2}\left(k_{n p} r\right) J_{n+1 / 2}\left(k_{r p} r^{\prime}\right)}{\left[J_{n+1 / 2}^{\prime}\left(k_{n p}\right)\right]^{2}} P_{n}(\cos \theta) \\
& \times P_{n}\left(\cos \theta^{\prime}\right) \exp \left(-k_{n p}^{2} t\right),
\end{aligned}
$$

where $k_{n p}$ are the positive zeros of $J_{n+1 / 2}(k)$. We label the right side of equation (4) as $F(T)$ and define the iteration scheme

$$
T_{j+1}=F\left(T_{j}\right) \text { for } j \geqslant 0 .
$$

Since the non-linear term $\exp (\alpha T /(\alpha+T))$ is bounded, an upper solution $\bar{T}$ can be constructed such that $T<\bar{T}$ for all $t$. Hence the operator $F(T)$ is compact. The sequence $\left\{F\left(T_{j}\right) \mid j \geqslant 0\right\}$ therefore has a convergent subsequence converging to a unique limit. Further, since the derivative of $\exp (\alpha T /(\alpha+T))$ with respect to $T$ is bounded, the initial value problem (1) and (2), and hence (4), has a 
unique solution (see [2]). To estimate the steady state solution of (1), or (4), we carry out the following asymptotic analysis for $t$ large. In what follows, we write $\phi(r, \theta, t)=0(\chi(\cdot))$ if there exists a constant $A$ such that $|\varphi|<A|\chi|$ for all values $r, \theta$ within the sphere and $t>0$. We write $\chi(\cdot)$ to emphasize that $\chi$ is a function of its argument only. If $\chi$ is a numerical constant, we shall write $\varphi(r, \theta, t)=$ $O(1)$. If we compare two numerical constants, $A=O(B)$ means that $A$ and $B$ are of comparable magnitude.

Let $Z$ be sufficiently large so that for $t-\tau \geqslant Z$, we have

$$
\begin{aligned}
G(P, Q, t-\tau) & \sim \frac{1}{2 \pi \sqrt{r r^{\prime}}} \frac{J_{1 / 2}\left(k_{01} r\right) J_{1 / 2}\left(k_{01} r^{\prime}\right)}{\left[J_{1 / 2}^{\prime}\left(k_{01} r\right)\right]^{2}} \exp \left(-k_{01}^{2}(t-\tau)\right) \\
& \equiv G_{01}(P, Q, t-\tau) .
\end{aligned}
$$

Here, we note that $k_{01}$ is the smallest number in the set $\left\{k_{n p}\right\}$, and $k_{01}=\pi$. Then, for $t-\tau \geqslant Z$, we have

$$
\begin{aligned}
T_{j+1} \sim & \delta \int_{0}^{t-z} \int_{D} G_{01}(P, Q, t-\tau) \exp \left(\frac{\alpha T_{j}(Q, \tau)}{\alpha+T_{j}(Q, \tau)}\right) d V_{Q} d \tau \\
& +\delta \int_{t-z}^{t} \int_{D} G(P, Q, t-\tau) \exp \left[\frac{\alpha T_{j}(Q, \tau)}{\alpha+T_{j}(Q, \tau)}\right] d V_{Q} d \tau \\
= & \delta \int_{0}^{t} \int_{D} G_{01}(P, Q, t-\tau) \exp \left[\frac{\alpha T_{j}(Q, \tau)}{\alpha+T_{j}(Q, \tau)}\right] d V_{Q} d \tau \\
& +\delta \int_{t-z}^{t} \int_{D}\left[G(P, Q, t-\tau)-G_{01}(P, Q, t-\tau)\right] \\
& \times \exp \left[\frac{\alpha T_{j}(Q, \tau)}{\alpha+T_{j}(Q, \tau)}\right] d V_{Q} d \tau \\
= & \delta \int_{Z}^{t} \int_{D} G_{01}(P, Q, t-\tau) \exp \left[\frac{\alpha T_{j}(Q, \tau)}{\alpha+T_{j}(Q, \tau)}\right] d V_{Q} d \tau \\
& +\delta \int_{0}^{z} \int_{D} G_{01}(P, Q, t-\tau) \exp \left[\frac{\alpha T_{j}(Q, \tau)}{\alpha+T_{j}(Q, \tau)}\right] d V_{Q} d \tau \\
& +\delta \int_{t-z}^{t} \int_{D}\left[G(P, Q, t-\tau)-G_{01}(P, Q, t-\tau)\right] \\
& \times \exp \left[\frac{\alpha T_{j}(Q, \tau)}{\alpha+T_{j}(Q, \tau)}\right] d V_{Q} d \tau .
\end{aligned}
$$


For $t \gg Z$, the second term on the right is $O\left(\exp \left(-\pi^{2}(t-z)\right)\right)$. The third term on the right is equal to

$$
\begin{aligned}
& \delta \sum_{\substack{n=0 \\
p=1}}^{\infty} \frac{(2 n+1) J_{n+1 / 2}\left(k_{n p} r\right) P_{n}(\cos \theta)}{2 \pi\left[J_{n+1 / 2}\left(k_{n p}\right)\right]^{2} k_{n p}^{2} \sqrt{r}}\left(1-\exp \left(-k_{n p}^{2} Z\right)\right) \\
& \quad \times \int_{D} J_{n+1 / 2}\left(k_{n p} r^{\prime}\right) P_{n}\left(\cos \theta^{\prime}\right) \frac{1}{\sqrt{r^{\prime}}} \exp \left(\frac{\alpha T_{j}(Q, \bar{\tau})}{\alpha+T_{j}(Q, \bar{\tau})}\right) d V_{Q} d \tau,
\end{aligned}
$$

where the prime after the summation sign means that the particular term with subscript $n=0, p=1$ is to be omitted, and $t-Z<\bar{\tau}<t$. To estimate the above, we observe that for $t \gg Z$ and $Z$ and $j$ sufficiently large, $T_{j}(Q, \bar{\tau})$ will be close to the steady state. In the steady state, $T$ is governed by the equation

$$
\nabla^{2} T=-\delta \exp ((\alpha T /(\alpha+T))) \text {, }
$$

with $T=0$ at $r=1$. Since the Laplacian is an intrinsic quantity not dependent on the coordinate system used, and since the function $\exp ((\alpha T /(\alpha+T)))$ does not depend explicitly on the spatial coordinates, rotation of the axes leaves equation (8) invariant. In spherical polar coordinates, we must have $\partial T / \partial \theta=0$ on the axis. This condition, together with the freedom to rotate axes, implies that $T(r, \theta, t)$ is a function of $r$ alone, as $t$ tends to infinity. If we then examine $T$ in terms of its eigenfunction expansion, we can deduce that the leading term is dominant (see Tam [6]). Thus, we have $T(Q, \bar{\tau}) \sim\left(M /(2 r)^{1 / 2}\right) J_{1 / 2}\left(\pi r^{\prime}\right)$ for some positive constant $M$. Because of its sole radial dependence, the asymptotic analysis of $T_{j+1}$ for the present case is the same as that for the case when $T$ is assumed to depend only on the radial distance for all $t>0$, as in [6]. The following results are therefore included only for the sake of completeness. For their derivation, the readers are referred to [6]. In approximating $T_{j+1}$, it was shown that we can use

$$
\begin{aligned}
T_{j+1} \sim & \frac{\delta \pi}{4 \sqrt{r^{\prime}}} J_{1 / 2}(\pi r) \int_{Z}^{t} \int_{D} \exp \left(-\pi^{2}(t-\tau)\right) \frac{J_{1 / 2}\left(\pi r^{\prime}\right)}{\sqrt{r^{\prime}}} \\
& \times \exp \left[\frac{\alpha T_{j}(Q, \tau)}{\alpha+T_{j}(Q, \tau)}\right] d V_{Q} d \tau .
\end{aligned}
$$

Now suppose, for $t>Z$, we have

$$
\frac{\sqrt{\pi}}{2} \int_{D} \frac{J_{1 / 2}\left(\pi r^{\prime}\right)}{\sqrt{r^{\prime}}} \exp \left[\frac{\alpha T_{j}(Q, \tau)}{\alpha+T_{j}(Q, \tau)}\right] d V_{Q}>K_{j}
$$

for some $j$, where $K_{j}$ is independent of $\tau$. Then there exists $Z_{j}>Z$ such that, for $t \gg Z_{j}$, we have

$$
T_{j+1}>\frac{\delta K_{j}}{2 \pi^{3 / 2} \sqrt{r}} J_{1 / 2}(\pi r)=\frac{\delta K_{j}}{\sqrt{2 \pi} \pi^{2}} \frac{\sin \pi r}{r} .
$$


Using the above representation for $T_{j+1}$, we can proceed to consider the next iteration. Suppose we have

$$
\frac{\sqrt{\pi}}{2} \int_{D} \frac{J_{1 / 2}\left(\pi r^{\prime}\right)}{\sqrt{r^{\prime}}} \exp \left[\frac{\alpha T_{j+1}(Q, \tau)}{\alpha+T_{j+1}(Q, \tau)}\right] d V_{Q} \geqslant K_{j+1} ;
$$

then we will have

$$
T_{j+2} \geqslant \frac{\delta K_{j+1}}{2 \pi^{3 / 2} \sqrt{r}} J_{1 / 2}(\pi r) .
$$

In this way, we generate a sequence of numbers $\left\{K_{i}\right\}, i=j, j+1, \ldots$ If, for a given $\delta$, we have $K_{j+1} \geqslant K_{j}$, then the sequence $\left\{K_{i}\right\}$ is monotone increasing. Since we know the solution for $T$ is bounded, $\left\{K_{i}\right\}$ tends to a limit. If the limit $K_{\infty}=O\left(e^{\alpha}\right)$, the solution of the initial value problem is super-critical.

To render the integral in (9) tractable, a number of approximations were made, and we obtained

$$
K_{j+1} \equiv \frac{4 \sqrt{2 \pi}}{A^{3}}\left\{(A-2) e^{A}+(A+2)\right\},
$$

where $A=\alpha v /(\alpha \pi \sqrt{2 \pi}+v)$ and $v=K_{j} \delta$. In Figure 1 we have plotted $K_{j+1}$ against $v$ for $\alpha=20$. It is clear that a comparison of $K_{j}$ with $K_{j+1}$ becomes a comparison of the straight line $v / \delta$ with $K_{j+1}$. Similar figures can be obtained for other values of $\alpha$.
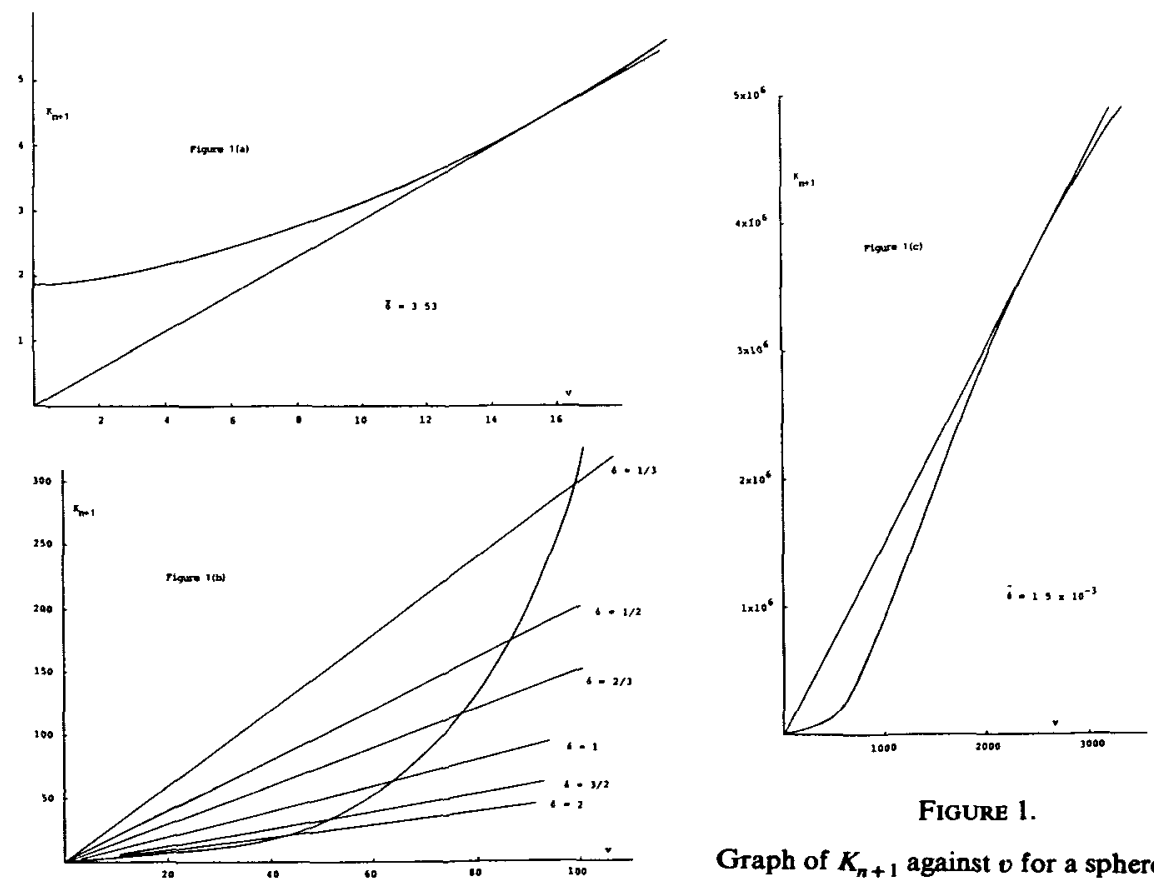

Figure 1.

Graph of $K_{n+1}$ against $v$ for a sphere. 


\section{The threshold phenomena}

We observe from Figure 1 that, when $\delta$ is sufficiently small, the straight line intersects $K_{j+1}$ at one point, where $K_{j+1}=O(1)$. When $\delta$ is increased beyond a certain value, say $\tilde{\delta}$, the straight line intersects $K_{j+1}$ at three points. When $\delta$ is further increased to be greater than $\bar{\delta}$, say, the number of intersections is reduced to one, where $K_{j+1}=O\left(e^{\alpha}\right)$. We derive the following information from Figure 1. When $\delta \geqslant \bar{\delta}$, the iteration scheme will settle to a steady state solution which is super-critical, regardless of the initial data. Thus $\bar{\delta}$ is a threshold value for the parameter $\delta$. When $\delta$ is less than $\tilde{\delta}$, the steady state solution is sub-critical. For $\delta$ between $\tilde{\delta}$ and $\bar{\delta}$, the initial data plays the deciding role. If we denote the coordinate of the middle intersection point of $v / \delta$ with $K_{j+1}$ by $\left(v^{*}, K^{*}\right)$, then, for a given $\delta$, if there is a $K_{j}$, for some $j$, such that $\delta K_{j} \geqslant v^{*}$, the steady state solution will be super-critical. As an illustration, we have obtained a few numbers graphically for $\alpha=20: \tilde{\delta}=1.5^{-1} \times 10^{-3}, \bar{\delta}=3.53$.

\begin{tabular}{|l|c|c|c|c|c|c|}
\hline$\delta$ & $1 / 3$ & $1 / 2$ & $2 / 3$ & 1 & $3 / 2$ & 2 \\
\hline$v^{*}$ & 99 & 87 & 77 & 64 & 51 & 44 \\
\hline
\end{tabular}

With the information obtained in the above, we are now in a position to answer the question set out in the Introduction. For fixed $\alpha$ and $\tilde{\delta}<\delta<\bar{\delta}$, to see whether a given initial $T_{0}(r, \theta)$ leads to a super-critical steady state solution, we calculate the inner product

$$
\frac{\sqrt{\pi}}{2} \int_{D} \frac{J_{1 / 2}(\pi r)}{\sqrt{r}} \exp \left[\frac{\alpha T_{0}(r, \theta)}{\alpha+T_{0}(r, \theta)}\right] d V_{Q}=K_{0}
$$

If the number so obtained is not less than $v^{*} / \delta$, the super-critical state will result. The inner product is readily calculated if $T_{0}(r, \theta)$ is as given in (3). We have

$$
\begin{aligned}
K_{0}= & \sqrt{2 \pi} \int_{0}^{1} \int_{0}^{\pi} r \sin \pi r \sin \theta \exp \left(\frac{\alpha T_{0}(r, \theta)}{\alpha+T_{0}(r, \theta)}\right) d r d \theta \\
\doteq & \sqrt{2 \pi} \int_{0}^{1} \int_{0}^{\pi} r \sin \pi r \sin \theta d r d \theta \\
& +\sqrt{2 \pi} \int_{r_{0}-r_{0}}^{r_{0}} \int_{0}^{\nu e} r \sin \pi r \sin \theta \exp \left(\frac{\alpha A}{\alpha+A}\right) d r d \theta \\
= & 2 \sqrt{\frac{2}{\pi}}+\frac{\sqrt{2 \pi}}{\pi^{2}} \exp \left(\frac{\alpha A}{\alpha+A}\right)(1-\cos \nu \varepsilon) \\
& \times\left[\sin \pi r_{0}-\pi r_{0} \cos \pi r_{0}-\sin \pi\left(r_{0}-\beta \varepsilon\right)+\pi\left(r_{0}-\beta \varepsilon\right) \cos \pi\left(r_{0}-\beta \varepsilon\right)\right] .
\end{aligned}
$$


If we use the fact that $\beta \varepsilon$ and $\nu \varepsilon$ are both small, we have

$$
\begin{aligned}
K_{0}= & 2 \sqrt{\frac{2}{\pi}}+\frac{1}{\pi^{2}} \sqrt{\frac{\pi}{2}} \exp \left(\frac{A}{\alpha+A}\right) \nu^{2} \beta \varepsilon^{3} \\
& \times\left\{\pi^{2} r_{0} \sin \pi r_{0}-\beta^{2} \varepsilon\left[\frac{\pi^{2}}{2} \sin \pi r_{0}+\frac{\pi^{3} r_{0}}{2} \cos \pi r_{0}\right]\right\} .
\end{aligned}
$$

Now, for $\alpha=20, \delta=1, v^{*}=64$. Thus, if $K_{0}>64$, the steady state solution will be super-critical. It is perhaps worth noting that if $\nu$ and $\beta$ are kept sufficient small, then $K_{0}$ cannot be made to be creater than $v^{*} / \delta$, no matter how large $A$ is. Indeed, for $A \rightarrow \infty$, we have

$$
K_{0} \sim 2 \sqrt{\frac{2}{\pi}}+\frac{1}{\pi^{2}} \sqrt{\frac{\pi}{2}} \nu^{2} \beta \varepsilon^{2}\left\{\pi^{2} r_{0} \sin \pi r_{0}-\beta^{2} \varepsilon\left[\frac{\pi^{2}}{2} \sin \pi r_{0}+\frac{\pi^{3} r_{0}}{2} \cos \pi r_{0}\right]\right\}
$$

Since $K_{0}$ depends on $r_{0}$, we make the following calculations to demonstrate this dependence. To have $K_{0}>64$, we need to have

$$
\nu^{2} \beta^{3} \varepsilon^{4}>47.6 \text { if } r_{0}=\beta \varepsilon
$$

and

$$
\nu^{2} \beta^{3} \varepsilon^{3}>31.70 \text { if } r_{0}=1 .
$$

Thus, no matter how hot the hot spot is, its extent must be sufficiently large for the super-critical state to result.

Another point of interest concerns the threshold values of $\delta$. For $\alpha=20$, the steady state solution is super-critical if $\delta>\bar{\delta}=3.53$, and subcritical if $\delta<\tilde{\delta}=$ $1.5^{-1} \times 10^{-3}$. Parks [5] has obtained $\delta_{\mathrm{cr}}=3.52$, so that $\bar{\delta}$ agrees well with $\delta_{\mathrm{cr}}$. To assess $\tilde{\delta}$, we note that in [7] Tam showed that, if $\delta<1.28 \times 10^{-5}\left(=\delta_{1}\right)$, then, regardless of the initial temperature, the steady state upper solution is sub-critical, and if $\delta<3.59 \times 10^{-3}\left(=\delta_{2}\right)$, the lower solution of the form $c\left(1-r^{2}\right)^{1.1}$ is sub-critical. Thus the value of $\delta$ lies between $\delta_{1}$ and $\delta_{2}$, as we would expect. Now the parameter $\tilde{\delta}$ is an extinction parameter. Unfortunately, the authors are not aware of published calculations on its magnitude, so that no comparison can be made. However, it must be said that the smallness of $\tilde{\delta}$ has rather serious implications. A system with a parameter $\delta$ much less than the critical value $(\sim 3)$ can become super-critical if it is subjected to heating by a sufficiently strong hot spot.

We conclude with the following remarks: (a) For different values of $\alpha$, the critical parameters for $\delta$ can be obtained from the graphs of $K_{j+1}$ against $v$, and the specification of the hot spot which determines sub- or super-criticality obtained from $K_{0}$. (b) Since our analysis leading to the expression $K_{0}$ hinges only on the assumption of rotational symmetry, that is $T=T(r, \theta, t)$, the result obtained can also be used for arbitrary $T_{0}(r, \theta)$. 


\section{Acknowledgment}

This research was supported by the Natural Science and Engineering Research Council of Canada under Grant A-5228 and A-9856. The authors are grateful to Dr. G. C. Wake, Victoria University of Wellington, New Zealand, for his interest and helpful comments which have substantially improved the presentation of this paper.

\section{References}

[1] T. Boddington, P. Gray and G. C. Wake, "Criteria for thermal explosions with and without reactant consumption", Proc. Roy. Soc. A 357 (1977), 403-422.

[2] C. Y. Chan, "A first initial boundary value problem for a semi-linear heat equation", SIAM J. Appl. Math. 22 (1972), 529-537.

[3] D. A. Frank-Kamenetskii, Diffusion and heat transfer in chemical kinetics (Translation ed. J. P. Appleton) (Plenum Press, New York, 1959).

[4] I. M. Gelfand, "Some problems in the theory of quasi-linear equations", AMS Translations Ser. 229 (1963), 295-381.

[5] J. R. Parks, "Criticality criteria for various configurations of a self-heating chemical as functions of activation energy and temperature of assembly", J. Chem. Phys. 34 (1961), 46-50.

[6] K. K. Tam, "On the influence of the initial data in a combustion problem", J. Austral. Math. Soc. B 22 (1980), 193-209.

[7] K. K. Tam, "On the disapperance of criticality in the theory of thermal ignition", ZAMP 31 (1980), 762-766.

\section{Mathematics Department}

McGill University

Montreal, Quebec

Canada

and

Mathematics Department

St. Mary's University

Halifax, Canada 